\title{
Localization of the antigenic sites of Newcastle disease virus nucleocapsid using a panel of monoclonal antibodies
}

\begin{abstract}
A panel of six monoclonal antibodies (mAbs) against the nucleocapsid (NP) protein of Newcastle disease virus (NDV) was produced by immunization of Balb/c mice with purified recombinant NP protein. Western Blot analysis showed that all the mAbs recognized linearized NP epitopes. Three different NP antigenic sites were identified using deleted truncated NP mutants purified from Escherichia coli. One of the antigenic sites was located at the C-terminal end (residues 441 to 489) of the NP protein. Two other antigenic sites were located within the N-terminal end (residues 26-121 and 122-375). This study demonstrates that the $\mathrm{N}$ - and $\mathrm{C}$-terminal ends of the NP proteins are responsible in eliciting immune response, thus it is most likely that these ends are exposed on the NP.
\end{abstract}

Keyword: Monoclonal antibody; Nucleocapsid; Antigenic sites; Newcastle disease virus 